\title{
Shape and Compliance of Endothelial Cells after Shear Stress In Vitro or from Different Aortic Regions: Scanning Ion Conductance Microscopy Study
}

\author{
Claire M. F. Potter ${ }^{1,2}$, Sophie Schobesberger ${ }^{1,2}$, Martina H. Lundberg ${ }^{1}$, Peter D. Weinberg ${ }^{2}$, Jane A. \\ Mitchell ${ }^{1 *}$, Julia Gorelik ${ }^{1 *}$
}

1 Cardiothoracic Pharmacology, Pharmacology and Toxicology and Functional Microscopy, Cardiovascular Sciences, National Heart and Lung Institute, London, United Kingdom, 2 Department of Bioengineering, Imperial College London, London, United Kingdom

\begin{abstract}
Objective: To measure the elongation and compliance of endothelial cells subjected to different patterns of shear stress in vitro, and to compare these parameters with the elongation and compliance of endothelial cells from different regions of the intact aorta.

Materials and Methods: Porcine aortic endothelial cells were cultured for 6 days under static conditions or on an orbital shaker. The shaker generated a wave of medium, inducing pulsatile shear stress with a preferred orientation at the edge of the well or steadier shear stress with changing orientation at its centre. The topography and compliance of these cells and cells from the inner and outer curvature of ex vivo porcine aortic arches were measured by scanning ion conductance microscopy (SICM).

Results: Cells cultured under oriented shear stress were more elongated and less compliant than cells grown under static conditions or under shear stress with no preferred orientation. Cells from the outer curvature of the aorta were more elongated and less compliant than cells from the inner curvature.

Conclusion: The elongation and compliance of cultured endothelial cells vary according to the pattern of applied shear stress, and are inversely correlated. A similar inverse correlation occurs in the aortic arch, with variation between regions thought to experience different haemodynamic stresses.
\end{abstract}

Citation: Potter CMF, Schobesberger S, Lundberg MH, Weinberg PD, Mitchell JA, et al. (2012) Shape and Compliance of Endothelial Cells after Shear Stress In Vitro or from Different Aortic Regions: Scanning Ion Conductance Microscopy Study. PLoS ONE 7(2): e31228. doi:10.1371/journal.pone.0031228

Editor: Shawn E. Bearden, Idaho State University, United States of America

Received September 22, 2011; Accepted January 4, 2012; Published February 16, 2012

Copyright: (C) 2012 Potter et al. This is an open-access article distributed under the terms of the Creative Commons Attribution License, which permits unrestricted use, distribution, and reproduction in any medium, provided the original author and source are credited.

Funding: British Heart Foundation Centre of Research Excellence at Imperial College London BHF RE/08/02 (http://www.bhf.org.uk). The funders had no role in study design, data collection and analysis, decision to publish, or preparation of the manuscript.

Competing Interests: The authors have declared that no competing interests exist.

* E-mail: j.gorelik@ic.ac.uk (JG); j.a.mitchell@ic.ac.uk (JAM)

\section{Introduction}

It has long been known that endothelial cells are rounded and randomly oriented when cultured under static conditions, but become elongated and aligned with the flow when exposed to a unidirectional shear stress [1]. Non-reversing pulsatile shear has a greater elongatory effect than steady shear of the same mean magnitude, reversing pulsatile shear has a lesser effect and oscillatory shear has no effect at all [2]. Endothelial cell border and nuclear elongation have been used to assess local shear stresses occurring in vivo and hence to explore the relation between shear and susceptibility to atherosclerosis [3,4]. It has been proposed that more rounded cells, found in areas prone to plaque formation and predicted to be exposed to low shear stress, are dysfunctional and thus much has been done to investigate the physical properties of cells from both susceptible and protected regions of the vasculature and identify the differences between them. Initial studies focused on assessment of cell nuclear elongation and alignment in vivo [5] whilst later work went on to focus on cell morphology both in vivo [6,7] and in vitro [1,8,9]. An increasing body of work suggests that cell morphology change is driven by cytoskeletal rearrangement $[10,11,12]$ though the full mechanisms behind the mechanotransduction of shear stress remain unclear.

One area of focus in the evaluation of cell structure is measurement of local cell mechanobiology. It is known that on exposure to shear stress endothelial cell movement is reduced whilst polymerisation of filamentous actin is increased and actin stress fibres form in alignment with the direction of flow. These stress fibres are thought to cause a fall in the compliance of the endothelial cell membrane [13]. Endothelial cell membrane compliance has been measured by a number of methods. Such methods include those exerting a pulling force such as use of optical tweezers, whereby a bead is trapped in position over a cell and manipulated to make contact with the cell and then repositioned pulling on the cell membrane, magnetic rheology, in which an integrin coated bead is bound to a cell and manipulated by magnets [14] and micropippete aspiration [13] whereby suction pressure is used to pull the cell surface up into a 
glass tube. Though these methods allow for application of a range of forces they are limited in their ability to study cells in their native context, often requiring cells to be in suspension and require direct and potentially damaging contact with the cell. Other methods such as atomic force microscopy where the cell is probed with a cantilever exert a force pushing into the cell [15] but again this level of contact could be potentially damaging giving rise to artefacts potentially influencing results. The majority of the above methods are also limited in their spatial or temporal abilities and can only record one feature at a time. With the development of Scanning Ion Conductance Microscopy (SICM), such restrictions can be avoided. By allowing scanning and mechanical testing with minimal invasiveness, SICM is ideal for the investigation of living cells, enabling accurate recording of cell properties in their native context and when grown in vitro $[16,17]$.

We recently characterised a model to subject endothelial cells to different patterns of shear stress [18]. By placing circular culture wells on an orbital shaker, cells at the edge of the well are exposed to shear that fluctuates in magnitude and has a preferred (tangential) orientation whilst cells in the centre of the well are exposed to shear of a uniform magnitude (with the same mean value as at the edge) that fluctuates between radial and tangential orientations. We showed that cells at the edge are more aligned than those at the centre [18]. Here we use this model in conjunction with SICM to investigate whether porcine aortic endothelial cells (PAEC) differ in elongation and compliance depending upon the shear profile they experience. We additionally investigated these properties in the inner and outer curvature of the ascending porcine aorta.

\section{Materials and Methods}

\section{Isolation and culture of porcine aortic endothelial cells (PAEC)}

Cells were isolated from the descending thoracic aortas of Landrace cross pigs (Fresh Tissue Supplies, East Sussex, UK) by collagenase digestion as described in the method of Bogle et al [19]. These aortas were collected in Hank's Balance Salt Solution (HBSS) supplemented with antibiotics (All Sigma UK), delivered within 24 hours of animal slaughter and stored at $4^{\circ} \mathrm{C}$ until use. All cells used in experiments were at passage 2 .

\section{Dissection of porcine aortas}

Porcine hearts with attached aortas were obtained directly from the abattoir (Cheale Meats, Essex, UK) within 4 hours of animal slaughter in antibiotic supplemented HBSS. Aortas were inspected for damage before use. The aorta was first removed from the heart before excision of squares of tissue, approximately $2.5 \mathrm{~cm} \times 2.5 \mathrm{~cm}$ in area, from regions of interest defined by anatomical landmarks. The 'outer curvature' section was taken approximately $0.5 \mathrm{~cm}$ proximal to the origin of the bracheocephalic artery and the inner curvature from a region approximately opposite this (Figure 1).

The tissue samples were stripped of excess connective tissue and washed in PBS before they were pinned to wax patches in dishes containing L15 medium without phenol red (Invitrogen, UK) for SICM.

\section{Scanning lon conductance microscopy (SICM)}

The SICM method has been described previously [17]. Briefly, the SICM probe consists of a glass nanopipette filled with electrolyte. The ion current will decrease the closer the pipette is brought to the sample surface until a predetermined level is reached and a feedback control stops the pipette from moving further. A plot of pipette position creates an image of the cell

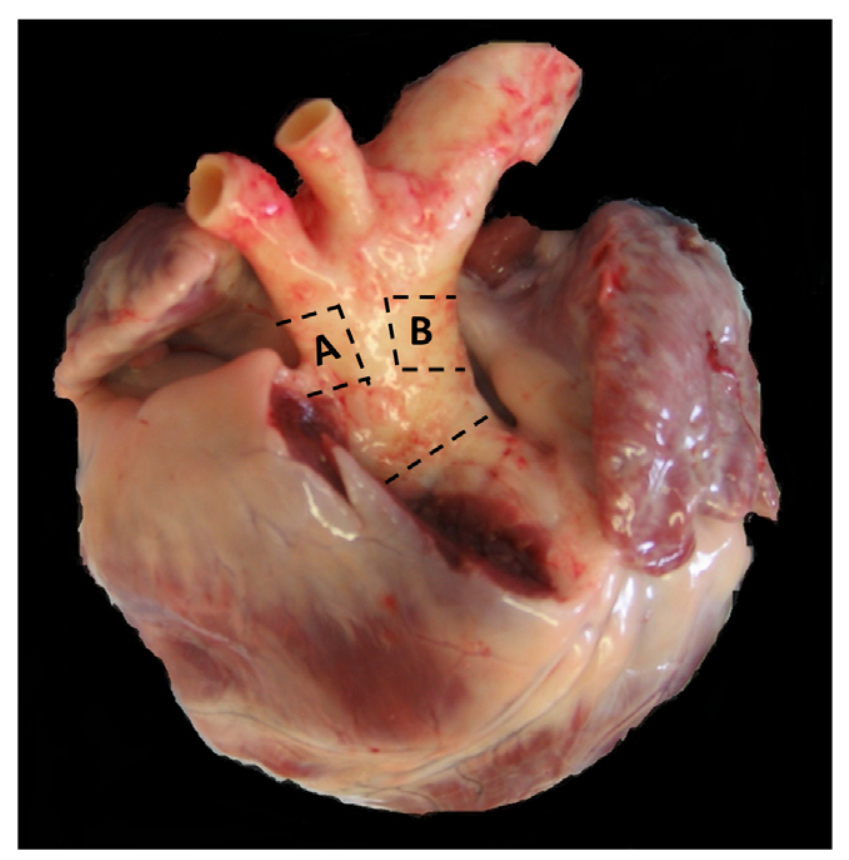

Figure 1. Image of a porcine heart and aorta illustrating the regions examined in this study. $A$ is a section of the outer curvature, and $B$ the inner curvature, of the ascending proximal aorta. doi:10.1371/journal.pone.0031228.g001

surface. To improve the reliability of the method we recently introduced hopping mode SICM [20].

Monolayers of PAEC grown with or without shear stress were scanned by SICM (Ionscope). Micropipettes for scanning were pulled using a Laser puller (Sutter Instruments, P-2000); they had a diameter of $500 \mathrm{~nm}$ and a resistance of $25 \mathrm{M} \Omega$. Topographic images were obtained by scanning an $80 \mu \mathrm{m} \times 80 \mu \mathrm{m}$ or $64 \mu \mathrm{m} \times 64 \mu \mathrm{m}$ area in hopping mode. Cultured cells and aortic segments were scanned for a maximum of 2 hours.

\section{Compliance measurements}

Using the principle described before [17], SICM was used to determine mechanical properties of cells by subjecting them to locally produced dynamic pressure (see figure 2).

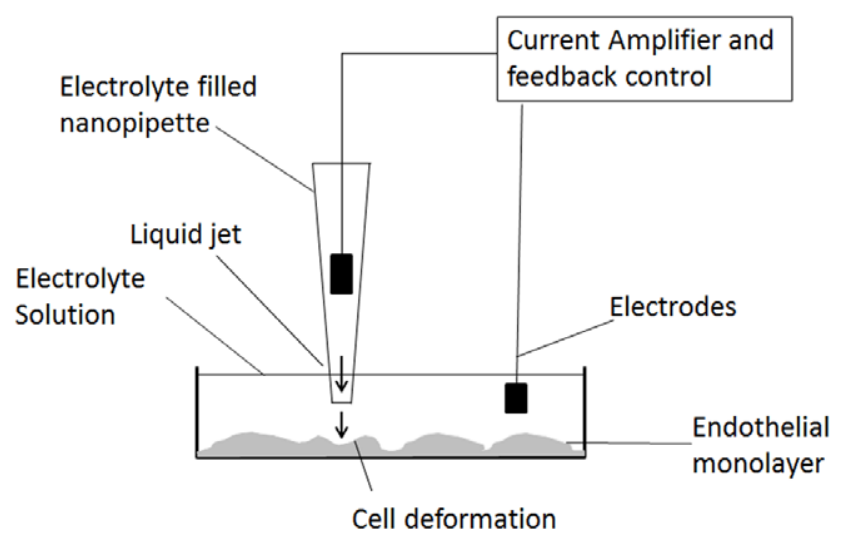

Figure 2. Schematic depiction of the SICM setup in a typical cell compliance experiment.

doi:10.1371/journal.pone.0031228.g002 
To calculate the compliance of single cells as result of local dynamic pressure, the resistance of the pipette $(\mathrm{R})$, the induced pressure and the resulting displacement of the cell surface were determined. Calculations were performed using the following mathematical formula:

$$
\mathrm{C}=\mathrm{d} / \mathrm{P} \times \sqrt{\mathrm{R}} / \sqrt{\mathrm{Ri}}
$$

where $\mathrm{C}=$ compliance $\mathrm{d}=$ displacement of the membrane $(\mu \mathrm{m})$; $\mathrm{P}=$ pressure $(\mathrm{kPa}) ; \mathrm{R}=$ actual pipette resistance $(\boldsymbol{\Omega}) ; \mathrm{Ri}=$ ideal pipette resistance $(\boldsymbol{\Omega})$.

The displacement measurement, $\mathrm{d}$, was obtained at maximal applied $\mathrm{P}$, which was usually in the range $30-40 \mathrm{kPa}$. Compliance measurements were calculated only where there was demonstrable recovery of the cell membrane to its original height.

\section{Calculation of index of elongation and data inclusion}

Measurements of elongation were obtained using ScanIC image (Ionscope, UK). Only complete cells with clear borders were included in the analysis. Images were excluded where the endothelial cell surface appeared damaged in any way. Length measurements were taken at the longest point of the cell and width values from the broadest part of the cell. Length was divided by width to obtain the index of elongation (Figure $3 \mathrm{~A}$, third image).

\section{Results}

SICM images revealed that PAEC grown under static conditions and in the centre of a culture well under shear had polygonal morphology whereas PAEC from the edge of the sheared well had an elongated and aligned appearance. Endothe-

\section{A}

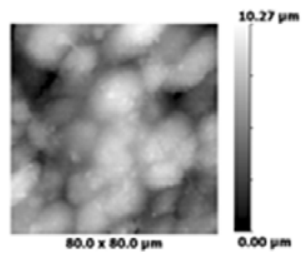

Inner curvature of ascending aortic arch

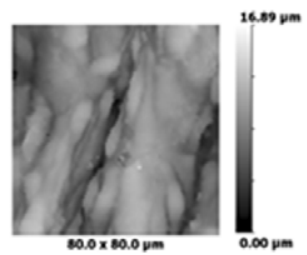

Outer curvature of ascending aortic arch

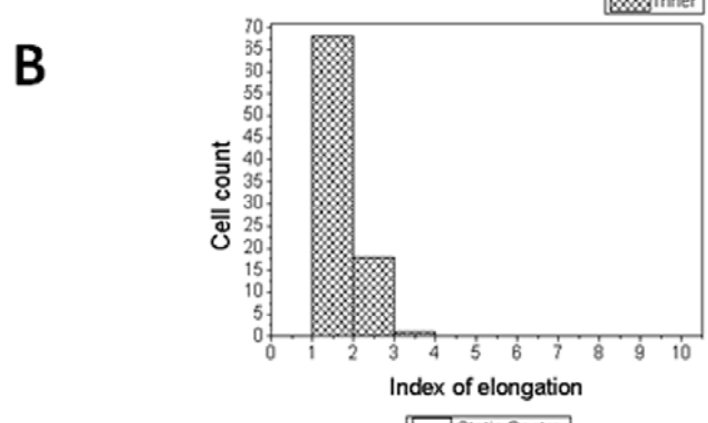

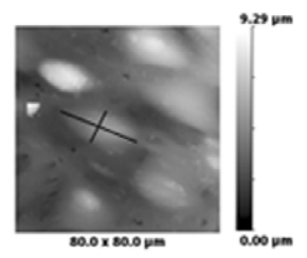

Cell exposed to directional shear stress (edge)

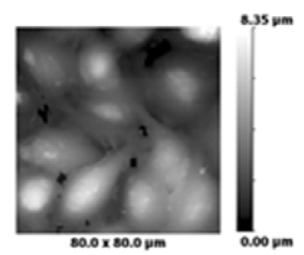

Cell exposed to non-directional shear stress (centre)

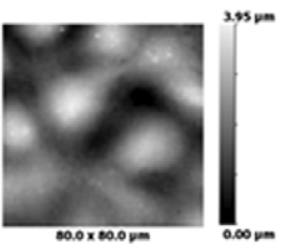

Cell grown under static conditions (control)
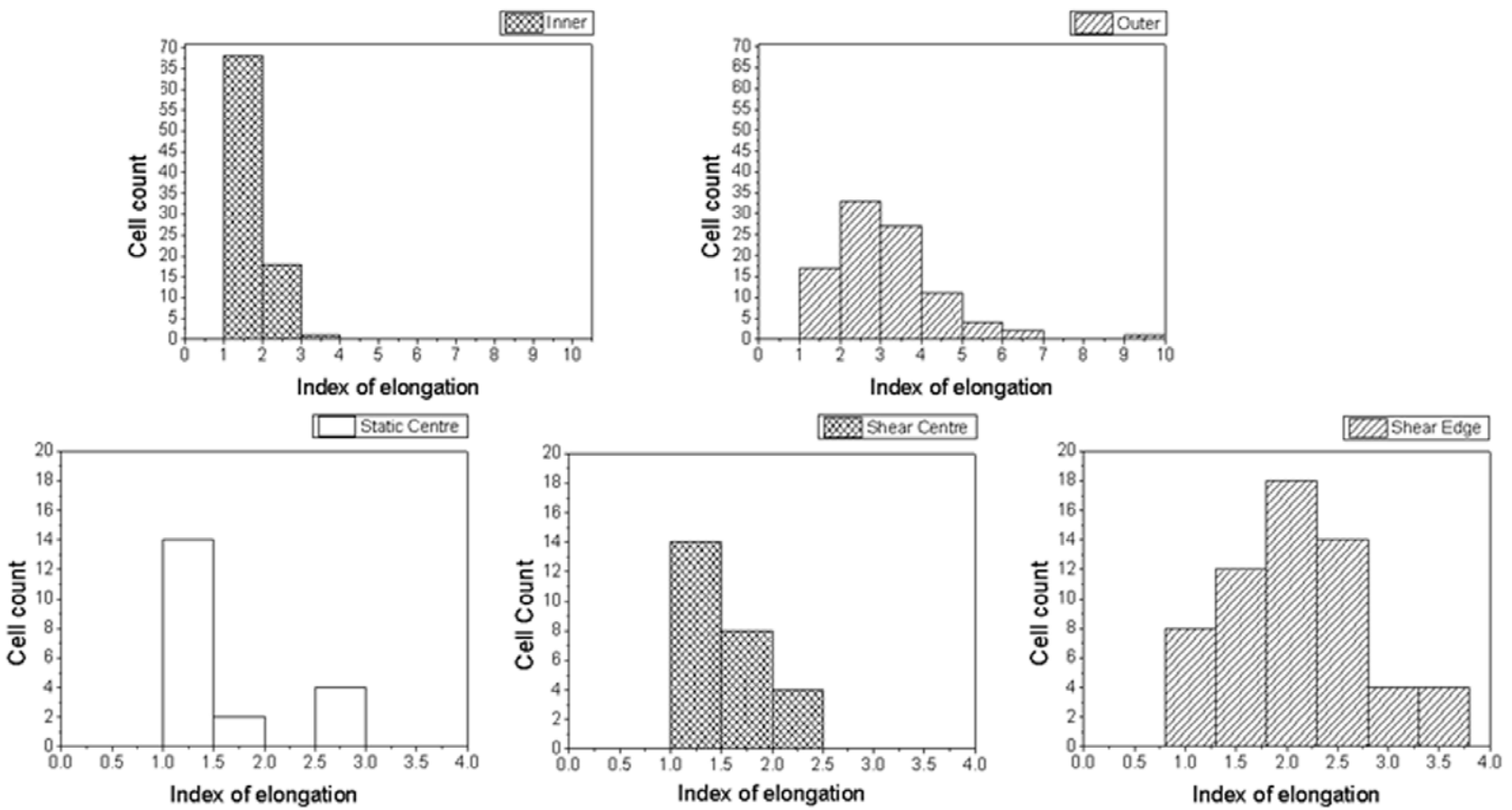

Figure 3. Representative SICM images from different parts of aorta and endothelial cells after shear stress. (A) Representative topographical SICM images of $80 \times 80 \mu \mathrm{m}$ regions of the inner and outer curvature regions of the ascending pig aorta and of PAEC cultured under static (control) or sheared conditions. The greyscale represents the height of the cells. Edge=a region near the edge of the well thought to experience directional pulsatile shear and centre $=\mathrm{a}$ region near the centre of the well thought to experience non-directional steady shear (B) Distribution of IE under all conditions studied. (Upper graphs- inner and outer parts of aorta; Bottom graphs-cell culture-static centre, shear centre and shear edge).

doi:10.1371/journal.pone.0031228.g003 
lial cells lining the outer curvature of the ascending porcine aorta were also elongated and aligned with each other, whilst cells from the inner curvature were more rounded and had no tendency for alignment (Figure 3A).

Cell morphology was quantified using the Index of Elongation (IE), a calculated length-to-width ratio generated by ScanIC Image. Cells at the sheared well edges had an average IE of 2.02, exceeding the average IE of 1.4 for cells grown in the centres of the same wells. Cells of the outer curvature of the pig aorta had an average IE of 2.61, exceeding that of PAEC grown under all conditions (Figure 3). The cells of the inner curvature of the aorta had an average ratio of 1.7. There was increased variability in alignment in cells from the native aorta as compared to cultured cells as might be expected based on differences between vessel geometries. There was a tendency for PAEC from the outer curvature to have a greater IE than PAEG from the inner curvature, many of which appeared only partially elongated or round (Figure 3B).

The SICM probe is a nanopipette that can produce a jet of buffer, and this allowed measurement of cell compliance by local application of a dynamic pressure (Figure 2). The compliance of cells in the outer curvature of the ascending aorta (0.012 \pm $0.002 \mu \mathrm{m} / \mathrm{kPa}$ ) was close to the compliance of PAEC cultured at the sheared well edge $(0.016 \pm 0.003 \mu \mathrm{m} / \mathrm{kPa})$. The compliance of cells in the inner curvature was higher $(0.027 \pm 0.008 \mu \mathrm{m} / \mathrm{kPa})$ and approached that of PAEC cultured in the shear well centre $(0.038 \pm 0.006 \mu \mathrm{m} / \mathrm{kPa})$. The compliance of cells from all regions of the well cultured under static conditions $(0.035 \pm 0.006 \mu \mathrm{m} / \mathrm{kPa})$ was nearly identical to those of PAEC cultured in the shear well centre (Figure 4A).

Figure 4B shows the relationship between index of elongation and compliance for endothelial cells from the in vitro shear stress model and the native aorta. For the range of compliances occupied by cells of both types, there was a remarkable agreement in the slope of the regression lines. Some compliance values measured from the least elongated cells in vitro substantially exceeded any compliances measured in the intact aorta; a large spread was seen in compliance values for these cells.

\section{Discussion}

Scanning ion conductance microscopy (SICM) was invented by Paul Hansma in the 1980s [21] and was later adopted to image and analyse the surface topography of live cells [20,22,23]. This was possible because SICM uses a nanopipette as a scanning probe which can be utilised to image cell surface structures with nanometre resolution without touching the cell [23]. Our laboratory further developed this system, introducing a plethora
A

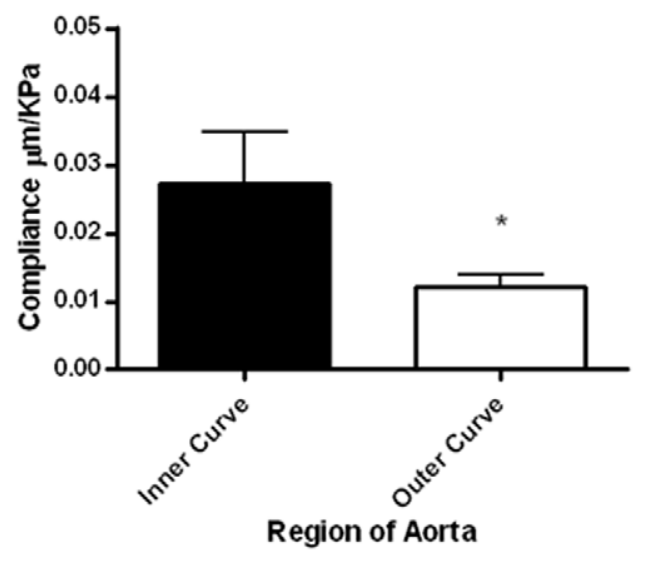

C

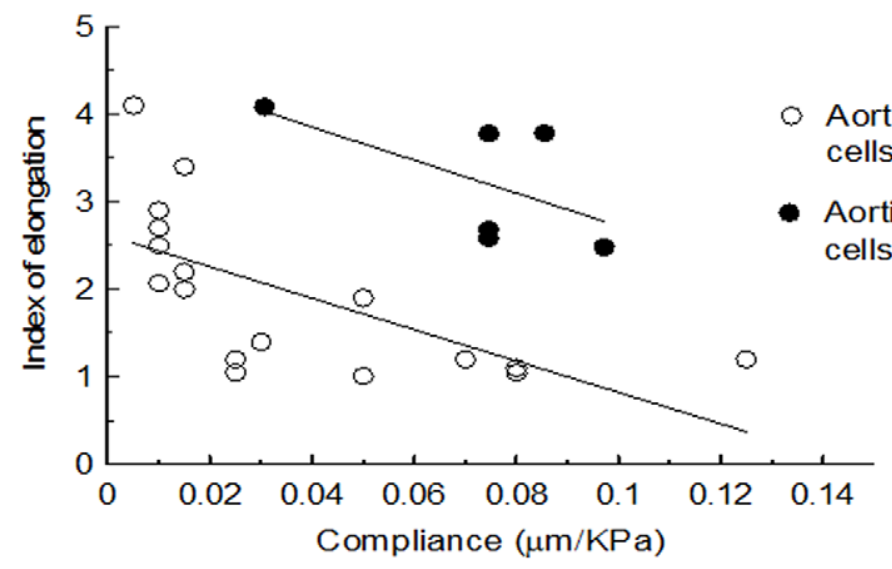

B

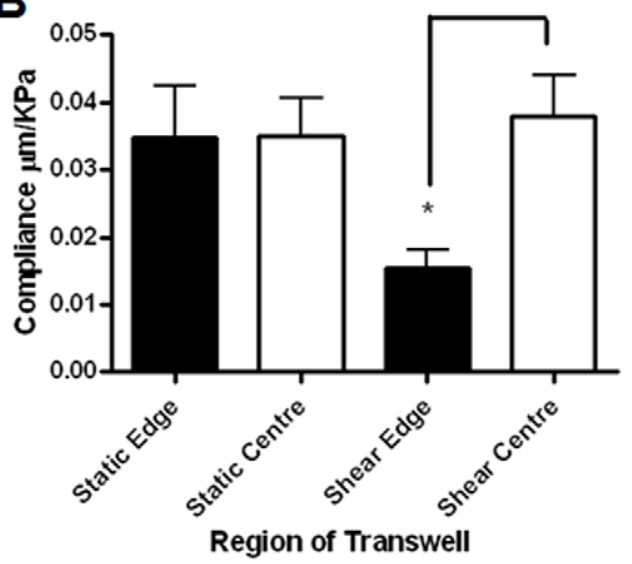

Aortic endothelial cells in culture

Aortic endothelial ells in-situ

Figure 4. Compliance measurements from different parts of aorta and endothelial cells after shear stress. (A); (B) Compliance of cells of the inner and outer curvatures of the ascending aorta and of cultured PAEC. Data were analysed by T-test. P $\leq 0.05$ was deemed significant and is indicated by *. (C) Relation between shape and compliance of cells in the aorta (linear correlation coefficient $\mathrm{R}=-0.56$ ) and PAEC (linear correlation coefficient $\mathrm{R}=-0.66$ ).

doi:10.1371/journal.pone.0031228.g004 
of associated methods [24,25,26]. Amongst other methods we developed SICM for measuring cell shape and volume. Recently, we have improved on the reliability and accuracy of SICM by introducing hopping probe ion conductance microscopy (HPICM) [20]. This allows us to obtain nanoscale resolution in highly convoluted live cell samples, such as whole tissue preparations e.g. the organ of Corti, without compromising the scan speed.

Endothelial cell elongation [2] and compliance [13] have both previously been shown to depend on applied shear stress in vitro. Here we used a recently developed cell culture model in conjunction with Scanning Ion Conductance Microscopy to measure elongation and compliance of individual cells exposed to pulsatile, oriented shear or non-pulsatile, non-oriented shear. We found a strong relation between the two cell properties with elongated cells showing reduced compliance. This in in agreement with previous studies carried out using AFM on cells that had experienced an acute application of shear stress [27].

\section{References}

1. Levesque MJ, Nerem RM (1985) The Elongation and Orientation of Cultured Endothelial Cells in Response to Shear Stress. Journal of Biomechanical Engineering 107: 341-347.

2. Helmlinger GG, R V, Schreck S, Nerem RM (1991) Effects of pulsatile flow on cultured vascular endothelial cell morphology. Journal of Biomechanical Engineering. pp 123-131.

3. Cybulsky MI, Iiyama K, Li H, Zhu S, Chen M, et al. (2001) A major role for VCAM-1, but not ICAM-1, in early atherosclerosis. The Journal of Clinical Investigation 107: 1255-1262.

4. Bond AR, Iftikhar S, Bharath AA, Weinberg PD (2011) Morphological Evidence for a Change in the Pattern of Aortic Wall Shear Stress With Age. Arteriosclerosis, Thrombosis, and Vascular Biology 31: 543-550.

5. Flaherty JT, Pierce JE, Ferrans VJ, Patel DJ, Tucker WK, et al. (1972) Endothelial Nuclear Patterns in the Canine Arterial Tree with Particular Reference to Hemodynamic Events. Circulation Research 30: 23-33.

6. Reidy MA, Levesque MJ (1977) A scanning electron microscopic study of arterial endothelial cells using vascular casts. Atherosclerosis 28: 463-470.

7. Goode TBDP, Reidy MA, Bowyer DE (1977) Aortic endothelial cell morphology observed in situ by scanning electron microscopy during atherogenesis in the rabbit. Atherosclerosis 27: 235-251.

8. C. F. Dewey J, Bussolari SR, M. A. Gimbrone J, Davies PF (1981) The Dynamic Response of Vascular Endothelial Cells to Fluid Shear Stress. Journal of Biomechanical Engineering 103: 177-185.

9. Eskin SG, Ives GL, McIntire LV, Navarro LT (1984) Response of cultured endothelial cells to steady flow. Microvascular Research 28: 87-94.

10. Malek A, Izumo S (1996) Mechanism of endothelial cell shape change and cytoskeletal remodeling in response to fluid shear stress. Journal of Cell Science 109: 713-726.

11. Helmke BP, Davies PF (2002) The Cytoskeleton Under External Fluid Mechanical Forces: Hemodynamic Forces Acting on the Endothelium. Annals of Biomedical Engineering 30: 284-296.

12. Mott RE, Helmke BP (2007) Mapping the dynamics of shear stress-induced structural changes in endothelial cells. American Journal of Physiology - Cell Physiology 293: C1616-C1626.

13. Sato M, Levesque MJ, Nerem RM (1987) Micropipette Aspiration of Cultured Bovine Aortic Endothelial-Cells Exposed to Shear-Stress. Arteriosclerosis 7: 276-286.

14. Feneberg W, Aepfelbacher M, Sackmann E (2004) Microviscoelasticity of the Apical Cell Surface of Human Umbilical Vein Endothelial Cells (HUVEC) within Confluent Monolayers. Biophysical Journal 87: 1338-1350.

15. Oberleithner H, Callies C, Kusche-Vihrog K, Schillers H, Shahin V, et al. (2009) Potassium softens vascular endothelium and increases nitric oxide release. Proceedings of the National Academy of Sciences 106: 2829-2834.
SICM also enabled us to make measurements of endothelial cell compliance from different regions of the aorta. We chose the inner and outer curvature of the ascending aorta; in mice these regions are atheroprone and atheroprotected, respectively [3], and experience different patterns of shear [28], although these properties may not hold for larger species. Differences between the regions of the aorta were similar to those found between the different culture conditions, and there was a similar relation between compliance and elongation. This new application of SICM presents exciting opportunities for future study.

\section{Author Contributions}

Conceived and designed the experiments: CMFP MHL PDW JAM JG. Performed the experiments: CMFP SS. Analyzed the data: CMFP SS JG. Contributed reagents/materials/analysis tools: JAM JG. Wrote the paper: CP PDW JAM JG.

16. Miragoli M, Moshkov A, Novak P, Shevchuk A, Nikolaev VO, et al. (2011) Scanning ion conductance microscopy: a convergent high-resolution technology for multi-parametric analysis of living cardiovascular cells. Journal of The Royal Society Interface 8: 913-925.

17. Sánchez D, Johnson N, Li C, Novak P, Rheinlaender J, et al. (2008) Noncontact Measurement of the Local Mechanical Properties of Living Cells Using Pressure Applied via a Pipette. Biophysical Journal 95: 3017-3027.

18. Potter CMF, Lundberg MH, Harrington LS, Warboys CM, Warner TD, et al. (2011) Role of Shear Stress in Endothelial Cell Morphology and Expression of Cyclooxygenase Isoforms. Arteriosclerosis, Thrombosis and Vascular Biology 31: $384-391$.

19. Bogle RG, Baydoun AR, Pearson JD, Mann GE (1996) Regulation of L-arginine transport and nitric oxide release in superfused porcine aortic endothelial cells. The Journal of Physiology 490: 229-241.

20. Novak P, Li C, Shevchuk AI, Stepanyan R, Caldwell M, et al. (2009) Nanoscale live-cell imaging using hopping probe ion conductance microscopy. Nature Methods 6: 279-281.

21. Hansma PK, Drake B, Marti O, Gould SA, Prater CB (1989) The scanning ionconductance microscope. Science 243: 641-643.

22. Korchev YE, Milovanovic M, Bashford CL, Bennett DC, Sviderskaya EV, et al. (1997) Specialized scanning ion-conductance microscope for imaging of living cells. Journal of Microscopy 188: 17-23.

23. Shevchuk AI, Frolenkov GI, Sanchez D, James PS, Freedman N, et al. (2006) Imaging proteins in membranes of living cells by high-resolution scanning ion conductance microscopy. Angewandte Chemie International Edition English 45: 2212-2216.

24. Sheikh Abdul Kadir SH, Miragoli M, Abu-Hayyeh S, Moshkov AV, Xie Q, et al. (2010) Bile acid-induced arrhythmia is mediated by muscarinic M2 receptors in neonatal rat cardiomyocytes. PLoS One 5: e9689.

25. Gorelik J, Gu Y, Spohr HA, Shevchuk AI, Lab MJ, et al. (2002) Ion channels in small cells and subcellular structures can be studied with a smart patch-clamp system. Biophysical Journal 83: 3296-3303.

26. Nikolaev VO, Moshkov A, Lyon AR, Miragoli M, Novak P, et al. (2010) Beta2adrenergic receptor redistribution in heart failure changes cAMP compartmentation. Science 327: 1653-1657.

27. Sato M, Nagayama K, Kataoka N, Sasaki M, Hane K (2000) Local mechanical properties measured by atomic force microscopy for cultured bovine endothelial cells exposed to shear stress. Journal of Biomechanics 33: 127-135.

28. Suo J, Ferrara DE, Sorescu D, Guldberg RE, Taylor WR, et al. (2007) Hemodynamic Shear Stresses in Mouse Aortas: Implications for Atherogenesis. Arteriosclerosis, Thrombosis and Vascular Biology 27: 346-351. 